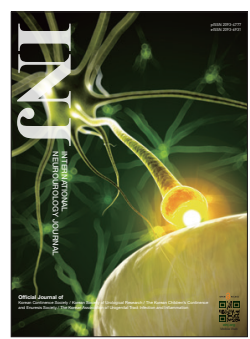

\title{
Research Goes on During Coronavirus Disease 2019 Pandemic
}

\author{
Su Jin Kim (id https://orcid.org/0000-0002-1917-2780 \\ Department of Urology, College of Medicine, Hallym Unversity, Kangdong Sacred Heart Hospital, Seoul, Korea \\ Email: hygeisujin@naver.com
}

Many changes have been made to adapt to the coronavirus disease 2019 (COVID-19) pandemic, and current clinical practice is also now substantially different compared to before COVID-19. Despite the difficulties induced by COVID-19, many clinical and basic researchers contributed to the last issue of the International Neurourology Journal (INJ) in 2021.

Older people and patients with neurological diseases are especially vulnerable to COVID-19; therefore, particular concern about their health is necessary. This issue of INJ offers articles presenting a comprehensive overview of aging and the lower urinary tract (LUT). Nishii [1] reviewed epidemiological perspectives on the aging LUT and discussed how frailty affects and deteriorates LUT symptoms (LUTS) in the older population. The management of LUTS due to aging involves special considerations, such as each individual patient's mental and functional characteristics as well as social status. Moreover, the economic burden associated with the treatment of LUTS in older people has been increasing in the aging society. Benign prostatic hyperplasia (BPH) is a common voiding problem in older men. Therefore, information about the prevalence of $\mathrm{BPH}$ and real-life practices helps to understand therapeutic trends in older men with BPH. Jo et al. [2] analyzed the characteristics of men with $\mathrm{BPH}$ over 60 years of age, and showed that medical therapy with an $\alpha$-blocker was the major treatment for $\mathrm{BPH}$, while holmium laser enucleation of the prostate has been increasing compared with transurethral resection of the prostate in Korea. The pathophysiology of aging-related LUTS has yet to be elucidated, although oxidative stress associated with ischemia/reperfusion injuries may be a possible underlying mechanism. Park et al. [3] showed that metformin and sildenafil ex- erted protective effects by decreasing reactive oxygen species through the activation of $\mathrm{p} 38$ mitogen-activated protein kinase, Bax, and Bcl-2, as well as by reducing inflammation through nuclear factor-kappa B inhibition.

Patients with different voiding problems often show similar LUTS, and sometimes LUTS in patients with the same diseases inducing voiding dysfunction are not identical. Thus, videourodynamic studies can be a valuable diagnostic method, especially in patients with spinal cord injuries [4]. Pediatric patients are more likely to feel uncomfortable in the clinic or hospital than adult patients. Therefore, mobile acoustic uroflowmetry (UFM) at home may be a better option in pediatric patients than conventional UFM in the hospital [5]. In addition to these attempts to improve the diagnostic process, treatment with greater benefits and fewer adverse events is important for healthy voiding and satisfactory quality of life. Studies on sacral and tibial nerve stimulation in patients with various LUTS in the present issue suggested that neural stimulation can be considered for patients in whom conservative and medical treatment have failed $[6,7]$.

At the beginning of 2021, I hoped that the COVID-19 pandemic would end this year. However, COVID-19 has continued more intensely than previously expected, and there have been many problems in our lives due to the effects of COVID-19. Nonetheless, the present pandemic is a chance for us to look back and reconsider our future. Moreover, new research studies are being conducted, and new attempts continue to be made to improve life throughout the world. Therefore, I expect that new findings during the COVID-19 pandemic will contribute to neurourological research. 
- Conflict of Interest: No potential conflict of interest relevant to this article was reported.

\section{REFERENCES}

1. Hisae Nishii. A review of aging and the lower urinary tract: the future of urology. Int Neurourol J 2021;25:273-84.

2. Jo JK, Shinn SH, Kim KS, Moon HS. Changes in prevalence and treatment pattern of benign prostatic hyperplasia in Korea. Int Neurourol J 2021;25:347-54.

3. Park JM, Shin JH, Yang SW, Lee JY, Lee CL, Lim JS, et al. Metformin and sildenafil attenuate inflammation and suppress apoptosis after ischemia/reperfusion injuries in rat urinary bladder. Int Neurourol J 2021;25:285-95.

4. Wang Z, Deng H, Li X, Liao L. The Video-urodynamic and electro- physiological characteristics in patients with traumatic spinal cord injury. Int Neurourol J 2021;25:327-36.

5. Lee DG, Gerber J, Bhatiaa V, Janzen N, Austin PF, Koh CJ, et al. A prospective comparative study of mobile acoustic uroflowmetry and conventional uroflowmetry. Int Neurourol J 2021;25:355-63.

6. Hernández-Hernández D, Padilla-Fernández B, Romera MC, Medler SH, Castro-Díaz D. Long-term outcomes of sacral nerve stimulation in pelvic floor dysfunctions. Int Neurourol J 2021;25: 319-26.

7. Girtner F, Fritsche HM, Zeman F, Huber T, Haider M, Pickl C, et al. Randomized crossover-controlled evaluation of simultaneous bilateral transcutaneous electrostimulation of the posterior tibial nerve during urodynamic studies in patients with lower urinary tract symptoms. Int Neurourol J 2021;25:337-46. 